\title{
Bridging Thrombolysis versus Direct Mechanical Thrombectomy in Stroke Due to Basilar Artery Occlusion
}

\author{
Isabel Siow,, a,* Benjamin Y.Q. Tan, ${ }^{\mathrm{a}, \mathrm{b}, *}$ Keng Siang Lee, ${ }^{\mathrm{c}}$ Natalie Ong, ${ }^{\mathrm{a}}$ Emma Toh, ${ }^{\mathrm{a}}$ Anil Gopinathan, ${ }^{\mathrm{a}, \mathrm{d}}$ \\ Cunli Yang, ${ }^{\mathrm{a}, \mathrm{d}}$ Pervinder Bhogal, ${ }^{\mathrm{e}}$ Erika Lam, ${ }^{\mathrm{f}}$ Oliver Spooner, ${ }^{\mathrm{f}}$ Lukas Meyer, ${ }^{\mathrm{g}}$ Jens Fiehler, ${ }^{\mathrm{g}}$ \\ Panagiotis Papanagiotou, ${ }^{\text {,i }}$ Andreas Kastrup, ${ }^{\mathrm{j}}$ Maria Alexandrou, ${ }^{\mathrm{h}}$ Seraphine Zubel, ${ }^{\mathrm{k}}$ Qingyu Wu, ${ }^{\mathrm{k}}$ \\ Anastasios Mpotsaris, ${ }^{\mathrm{k}}$ Volker Maus, ${ }^{\mathrm{l}}$ Tommy Anderson, ${ }^{\mathrm{m}, \mathrm{n}, \mathrm{o}}$ Vamsi Gontu, ${ }^{\mathrm{m}, \mathrm{n}}$ Fabian Arnberg, ${ }^{\mathrm{o}}$ \\ Tsong Hai Lee, ${ }^{\mathrm{p}}$ Bernard P.L. Chan, ${ }^{\mathrm{b}}$ Raymond C.S. Seet, ${ }^{\mathrm{b}}$ Hock Luen Teoh, ${ }^{\mathrm{b}}$ Vijay K. Sharma, ${ }^{\mathrm{a}, \mathrm{b}}$ \\ Leonard L.L. Yeo ${ }^{\mathrm{a}, \mathrm{b}}$ \\ a Yong Loo Lin School of Medicine, National University of Singapore, Singapore \\ ${ }^{b}$ Division of Neurology, Department of Medicine, National University Health System, Singapore \\ 'Bristol Medical School, University of Bristol, Bristol, UK \\ ${ }^{\mathrm{d}}$ Division of Interventional Radiology, Department of Diagnostic Imaging, National University Health System, Singapore \\ eDepartment of Interventional Neuroradiology, The Royal London Hospital, Barts NHS Trust, London, UK \\ fStroke Department, The Royal London Hospital, Barts NHS Trust, London, UK \\ ${ }^{9}$ Department of Diagnostic and Interventional Neuroradiology, University Medical Center Hamburg-Eppendorf, Hamburg, Germany \\ ${ }^{h}$ Department of Diagnostic and Interventional Neuroradiology, Hospital Bremen-Mitte, Bremen, Germany \\ 'First Department of Radiology, School of Medicine, National \& Kapodistrian University of Athens, Athens, Greece \\ 'Department of Neurology, Hospital Bremen-Mitte, Bremen, Germany \\ kDepartment of Neuroradiology, University Hospital Magdeburg, Magdeburg, Germany \\ Institute of Diagnostic and Interventional Radiology, Neuroradiology and Nuclear Medicine, University Hospital Knappschaftskrankenhaus \\ Bochum, Bochum, Germany \\ ${ }^{m}$ Department of Neuroradiology, Karolinska University Hospital, Stockholm, Sweden \\ ${ }^{n}$ Department of Clinical Neuroscience, Karolinska Institutet, Stockholm, Sweden \\ ${ }^{\circ}$ Department of Medical Imaging, AZ Groeninge, Kortrijk, Belgium \\ ${ }^{p}$ Department of Neurology, Linkou Chang Gung Memorial Hospital, Taoyuan, Taiwan
}

Background and Purpose Mechanical thrombectomy (MT) is an effective treatment for patients with basilar artery occlusion (BAO) acute ischemic stroke. It remains unclear whether bridging intravenous thrombolysis (IVT) prior to MT confers any benefit. This study compared the outcomes of acute BAO patients who were treated with direct MT versus combined IVT plus MT.

Methods This multicenter retrospective cohort study included patients who were treated for acute BAO from eight comprehensive stroke centers between January 2015 and December 2019. Patients received direct MT or combined bridging IVT plus MT. Primary outcome was favorable functional outcome defined as modified Rankin Scale 0-3 measured at 90 days. Secondary outcome measures included mortality and symptomatic intracranial hemorrhage (sICH).

Results Among 322 patients, 127 (39.4\%) patients underwent bridging IVT followed by MT and $195(60.6 \%)$ underwent direct MT. The mean \pm standard deviation age was $67.5 \pm 14.1$ years, $64.0 \%$ were male and median National Institutes of Health Stroke Scale was 16 (interquartile range, 8 to 25). At 90-day, the rate of favorable functional outcome was similar between the bridging IVT and direct MT groups (39.4\% vs. $34.4 \%, P=0.361)$. On multivariable analyses, bridging IVT was not as-
Correspondence: Leonard LL. Yeo Division of Neurology, Department of Medicine, National University Health System, 5 Lower Kent Ridge Rd, 119074 Singapore

Tel: +65-9061-6139

Fax: +65-6777-8065

E-mail: leonard_II_yeo@nuhs.edu.sg https://orcid.org/0000-0002-4249-0402

Received: June 9, 2021

Revised: August 13, 2021

Accepted: October 7, 2021

*These authors contributed equally to the manuscript as first author. 
sociated with favorable functional outcome, mortality or sICH. In subgroup analyses, patients with underlying atherosclerosis treated with bridging IVT compared to direct MT had a higher rate of favorable functional outcome at 90 days (37.2\% vs. 15.5\%, $P=0.013)$.

Conclusions Functional outcomes were similar in BAO patients treated with bridging IVT versus direct MT. In the subgroup of patients with underlying large-artery atherosclerosis stroke mechanism, bridging IVT may potentially confer benefit and this warrants further investigation.

Keywords Thrombolytic therapy; Thrombectomy; Stroke; Vertebrobasilar insufficiency; Basilar artery

\section{Introduction}

Acute basilar artery occlusion (BAO) is a cerebrovascular emergency that still causes devastating rates of morbidity and mortality despite the advances in acute ischemic stroke (AIS) care. $^{1,2}$ In some cases, basilar artery strokes can result in locked-in syndrome with quadriplegia and anarthria. ${ }^{3}$ An effective treatment modality for AIS due to large-vessel occlusion (LVO) is mechanical thrombectomy (MT). ${ }^{4-10}$ Administration of bridging intravenous thrombolysis (IVT) prior to MT is also recommended for most patients with LVO, pending results of several randomized controlled trials. ${ }^{11-15}$ The aim of IVT is to aid in recanalization of the culprit blood vessel and establish reperfusion, as well as to ameliorate any residual distal thrombi post$\mathrm{EVT}$, but this is balanced against the risk of hemorrhagic complications. ${ }^{16-18}$

Pooled analyses from several studies provide conflicting results regarding the efficacy of bridging IVT, with some studies reporting equivalent effects achieved in bridging IVT and direct $\mathrm{MT}_{1}{ }^{19-22}$ and others reporting an advantage with bridging treatment. ${ }^{23}$ However, most of these analyses included anterior circulation strokes. ${ }^{24}$ In patients with BAO who undergo MT, it remains to be elucidated whether prior treatment with bridging IVT is beneficial. An optimal clinical management protocol in patients with $\mathrm{BAO}$ has yet to be derived. ${ }^{25}$

We therefore performed a multicenter retrospective cohort study conducted across comprehensive stroke centers in Europe and Asia to determine whether outcomes of direct MT alone would be comparable to bridging IVT plus MT treatment in patients with BAO AIS.

\section{Methods}

\section{Ethics and standard protocol approvals}

This retrospective cohort study was approved by the Institutional Review Boards (IRB) of each respective institution. Waiver of individual participant consent was granted.

\section{Patients and treatment}

Consecutive patients from eight comprehensive stroke centers across five countries (Supplementary Table 1) with acute BAO who underwent MT between January 2015 and December 2019 were included. Of these, patients with a premorbid modified Rankin Scale $(m R S) \geq 2$ were excluded from this study. Prior to MT, all patients underwent baseline neurovascular imaging with computed tomography (CT) and CT angiography. Patients were considered for bridging thrombolysis if they presented within 4.5 hours of symptom onset, if they had no contraindications, and at the final discretion of the treating stroke neurologist. ${ }^{26}$ In each institution, IVT was administered by an accredited neurologist at a dose of $0.9 \mathrm{mg} / \mathrm{kg}$.

At all centers, patients were considered eligible for MT if the procedure could be initiated within 24 hours of the time of stroke onset and had an angiographically confirmed occlusion in the basilar artery. Patients were excluded from thrombectomy when pre-treatment imaging revealed extensive ischemic changes in the brainstem, or if the stroke was considered mild, based on an admission National Institutes of Health Stroke Scale (NIHSS) score of 3 or less. There was no upper limit on either the age of the patient or admission NIHSS score for inclusion across all centers.

Clinical monitoring was performed in either the intensive care unit or high dependency unit setting during the initial acute episode. All patients were managed according to international guidelines for the management of AIS. ${ }^{27}$ Participating stroke centers employed either a stent-retriever, direct aspiration catheter, or combined stent-retriever and aspiration via intermediate catheter approach from the start of the procedure. Patients who required intracranial stenting and intra-arterial thrombolysis were excluded from this study. Follow-up CT scan was performed at around 24 hours after initial treatment.

\section{Data collection}

The following epidemiological information was extracted from patient records: age, sex, race, and smoking history. Comorbidi- 
ties studied included hypertension, hyperlipidaemia, diabetes mellitus, atrial fibrillation, and previous ischemic stroke. The mechanism of ischemic stroke was defined according to the Trial of ORG 10172 in Acute Stroke Treatment (TOAST) classification, ${ }^{28}$ NIHSS score on admission and the time from stroke onset to groin puncture were extracted.

\section{Primary and secondary outcomes}

The primary outcome measure was a favorable functional outcome defined as a mRS $\leq 3$ after 90 days. ${ }^{2}$ Secondary measures of outcome were in-hospital mortality, good functional outcome defined as a $m R S \leq 2$ after 90 days, favorable mRS of $\leq 3$ upon discharge, symptomatic intracranial hemorrhage $(\mathrm{sICH})$ and subarachnoid hemorrhage (SAH). Reperfusion was assessed using post-procedure angiography, and successful reperfusion was defined as having a $2 b-3$ flow as calculated using the modified Thrombolysis in Cerebral Infarction (mTICl) scale. ${ }^{29}$ Additionally, a subset of patients who achieved $\mathrm{mTICl} 2 \mathrm{c}$ and 3 grades reperfu- sion was also tabulated. $\mathrm{sICH}$ was defined by the presence of parenchymal hemorrhage with an increase in NIHSS by $\geq 4$ points within 24 hours of revascularization according to the modified Safe Implementation of Thrombolysis in Stroke-Monitoring Study (SITS-MOST) criteria. Any SAH in the 24 hours post-thrombectomy CT imaging scans was included in the definition. ${ }^{30,31}$

\section{Statistical analysis}

Numeric variables were first tested for normality with the Shapiro-Wilk test. A Student's t-test was used for normally distributed data and a Mann-Whitney $\mathrm{U}$ test for non-normally distributed data. Categorical variables were compared using a Pearson chi-square test, with computation of Wald and score 95\% confidence interval (Cl) for the incidence odds ratio (OR). Subsequently, multivariable logistic regression was carried out to identify predictors for primary and secondary outcome measures. In addition, the following exploratory subgroup analyses were performed: (1) age 75 years or younger vs. older

Table 1. Baseline characteristics of study population $(n=322)$

\begin{tabular}{|c|c|c|c|c|}
\hline Characteristic & Total $(n=322)$ & Bridging IVT $(n=127)$ & Direct MT ( $n=195)$ & $P$ \\
\hline Age (yr) & $67.5 \pm 14.1$ & $69.4 \pm 14.0$ & $66.3 \pm 14.0$ & 0.054 \\
\hline Sex & & & & 0.050 \\
\hline Male & $206(64.0)$ & $73(57.5)$ & $133(68.2)$ & \\
\hline Female & $116(36.0)$ & $54(42.5)$ & $62(31.8)$ & \\
\hline Country & & & & - \\
\hline Germany & $138(42.9)$ & $65(51.2)$ & $73(37.4)$ & \\
\hline United Kingdom & $10(3.1)$ & $2(1.6)$ & $8(4.1)$ & \\
\hline Singapore & $67(20.8)$ & $33(26.0)$ & $34(17.4)$ & \\
\hline Taiwan & $46(14.3)$ & $7(5.5)$ & $39(20.0)$ & \\
\hline Sweden & $61(18.9)$ & $20(15.7)$ & $41(21.0)$ & \\
\hline Race & & & & 0.275 \\
\hline Caucasian & $209(64.9)$ & $87(68.5)$ & $122(62.6)$ & \\
\hline Asian & $113(35.1)$ & $40(31.5)$ & $73(37.4)$ & \\
\hline Hypertension & $198 / 260$ (76.2) & $88 / 106$ (83.0) & $110 / 154(71.4)$ & 0.031 \\
\hline Hyperlipidaemia & $83 / 256$ (32.4) & 38/103 (36.9) & $45 / 153(29.4)$ & 0.210 \\
\hline Diabetes mellitus & $75 / 260(28.8)$ & $31 / 106$ (29.2) & 44/154 (28.6) & 0.909 \\
\hline Atrial fibrillation & $77 / 260$ (29.6) & $27 / 106(25.5)$ & $50 / 154(32.5)$ & 0.225 \\
\hline Previous stroke & $34 / 215$ (15.8) & $16 / 86(18.6)$ & $18 / 129(14.0)$ & 0.360 \\
\hline TOAST classification & & & & 0.410 \\
\hline Large-artery atherosclerosis & $101(31.4)$ & $43(33.9)$ & $58(29.7)$ & \\
\hline Cardioembolic & $137(42.5)$ & $47(37.0)$ & $90(46.2)$ & \\
\hline Stroke of other determined aetiology & $12(3.7)$ & $6(4.7)$ & $6(3.1)$ & \\
\hline Stroke of undetermined aetiology & $72(22.4)$ & $31(24.4)$ & $41(21.0)$ & \\
\hline Admission NIHSS & $16(8-25)$ & $14(8-22)$ & $17(8-26)$ & 0.092 \\
\hline Time from stroke onset to groin puncture (min) & $270(180-420)$ & $240(180-300)$ & $330(180-518)$ & 0.002 \\
\hline Time from groin puncture to reperfusion (min) & $60(30-90)$ & $60(30-82)$ & $60(30-90)$ & 0.670 \\
\hline
\end{tabular}

Values are presented as mean \pm standard deviation, number (\%), or median (interquartile range).

IVT, intravenous thrombolysis; MT, mechanical thrombectomy; TOAST, Trial of Org 10172 in Acute Stroke Treatment; NIHSS, National Institutes of Health Stroke Scale. 
than 75 years; (2) male vs. female sex; and (3) TOAST mechanism of large-artery atherosclerosis (LAA) vs. non-LAA strokes. $P$ values $<0.05$ were considered statistically significant. The selection of variables for multivariable analyses was decided $a$ priori based on previously known factors from the literature that determine functional outcome after MT. Unknown or missing values were excluded from the denominator when calculating proportions in the study cohort. All statistical analysis was performed using SPSS version 26 (IBM Co., Armonk, NY, USA).

\section{Results}

\section{Baseline characteristics}

A total of 322 patients were included in the study. Of these patients, 127 (39.4\%) underwent bridging IVT prior to MT while 195 (60.6\%) underwent direct MT. The baseline characteristics of the bridging IVT group and direct MT group were comparable, with similar age, race, and comorbidity profile. Notably, there were more males in the direct MT group (68.2\%) compared to the bridging IVT group (57.5\%) $(P=0.050)$. In accor-

Table 2. Comparison of outcomes between direct MT and bridging IVT groups ( $n=322)$

\begin{tabular}{|c|c|c|c|c|}
\hline Outcome & Total $(n=322)$ & Bridging IVT $(n=127)$ & Direct MT $(n=195)$ & $P$ \\
\hline \multicolumn{5}{|l|}{ Primary outcome } \\
\hline 90-Day mRS & & & & 0.361 \\
\hline 0-3 (favorable) & $117(36.3)$ & $50(39.4)$ & $67(34.4)$ & \\
\hline 4-6 (unfavorable) & $205(63.7)$ & 77 (60.6) & $128(65.6)$ & \\
\hline \multicolumn{5}{|l|}{ Secondary outcomes } \\
\hline In-hospital mortality & & & & 0.226 \\
\hline Survival & $245(76.1)$ & $102(80.3)$ & $143(73.3)$ & \\
\hline Death & 77 (23.9) & 25 (19.7) & $52(26.7)$ & \\
\hline 90-Day mRS & & & & 0.593 \\
\hline $0-2$ (good) & $91(28.3)$ & $38(29.9)$ & $53(27.2)$ & \\
\hline $3-6$ (poor) & $231(71.7)$ & $89(70.1)$ & $142(72.8)$ & \\
\hline Discharge $\mathrm{mRS}$ & & & & 0.047 \\
\hline 0-3 (favorable) & $73 / 261(28.0)$ & $37 / 107$ (34.6) & $36 / 154(23.4)$ & \\
\hline 4-6 (unfavorable) & 188/261 (72.0) & 70/107 (65.4) & $118 / 154(76.6)$ & \\
\hline $\mathrm{mTICl}$ post-treatment & & & & 0.110 \\
\hline $0-2 a$ (poor) & $45 / 320(14.1)$ & $13 / 127(10.2)$ & $32 / 193(16.6)$ & \\
\hline $2 b-3$ (good) & $275 / 320$ (85.9) & $114 / 127$ (89.8) & 161/193 (83.4) & \\
\hline $\mathrm{mTICl}$ post-treatment & & & & 0.275 \\
\hline $0-2 b$ (poor) & $120 / 320(37.5)$ & 43/127 (33.9) & 77/193 (39.9) & \\
\hline $2 c-3$ (good) & $200 / 320(62.5)$ & $84 / 127(66.1)$ & 116/193 (60.1) & \\
\hline sICH 24-hour post-intervention & $14 / 299(4.7)$ & $6 / 119(5.0)$ & 8/180 (4.4) & 0.811 \\
\hline Subarachnoid hemorrhage & 9/264 (3.4) & 3/99 (3.0) & 6/165 (3.6) & 1.000 \\
\hline
\end{tabular}

Values are presented as number (\%).

MT, mechanical thrombectomy; IVT, intravenous thrombolysis; mRS, modified Rankin Scale; mTICl, modified Thrombolysis in Cerebral Infarction; sICH, symptomatic intracranial hemorrhage.

Table 3. Comparison of outcomes between bridging IVT and direct MT groups

\begin{tabular}{lcc}
\hline Outcome & $\mathrm{OR}(95 \% \mathrm{Cl})$ & $P$ \\
\hline 90-Day mRS & $1.21(0.67-2.18)$ & 0.524 \\
Discharge mRS & $1.60(0.82-3.10)$ & 0.169 \\
In-hospital mortality & $0.74(0.38-1.45)$ & 0.383 \\
Post-treatment mTICl 2b-3 & $2.33(0.98-5.53)$ & 0.056 \\
sICH 24-hour post-intervention & $1.65(0.49-5.59)$ & 0.422 \\
Subarachnoid hemorrhage & $0.96(0.20-4.54)$ & 0.955
\end{tabular}

Adjusted for age, sex, National Institutes of Health Stroke Scale and time from stroke onset to groin puncture.

$\mathrm{IVT}$, intravenous thrombolysis; $\mathrm{MT}$, mechanical thrombectomy; OR, odds ratio; $\mathrm{Cl}$, confidence interval; $\mathrm{mRS}$, modified Rankin Scale; mTICl, modified Thrombolysis in Cerebral Infarction; sICH, symptomatic intracranial hemorrhage. 
dance with TOAST classification, 101 patients (31.4\%) had underlying LAA and 137 patients (42.5\%) had stroke due to cardioembolism. There was a homogenous distribution of stroke mechanisms in the bridging IVT group and the direct MT group (Table 1).

Stroke severity as measured by median NIHSS was 16 on admission, and was marginally lower in the bridging IVT group compared to the direct MT group (14 vs. 17, $P=0.092$ ). Median time from stroke onset to groin puncture was longer in the direct MT group (median, 330 minutes; interquartile range [IQR], 180 to 518), compared to the bridging thrombolysis group (median, 240 minutes; IQR, 180 to 300; $P=0.002$ ). Median time from groin puncture to reperfusion was similar between both groups (Table 1).

\section{Primary outcome}

The rate of favorable functional outcome at 90 days was similar between the direct MT and bridging IVT groups (34.4\% vs. 39.4\%, $P=0.361$ ) (Table 2). After adjustment for age, sex, NIHSS, and time from stroke onset to groin puncture in the multivariable model, bridging IVT was not significantly associated with favorable functional outcome at 90 days $(\mathrm{OR}, 1.21 ; 95 \%$ $\mathrm{Cl}, 0.67$ to $2.18 ; P=0.524$ ) (Table 3).

\section{Secondary outcomes}

Secondary outcomes were largely similar between the direct MT group and the bridging IVT group, with the exception of discharge $\mathrm{mRS}$ (Table 2). The rate of favorable functional outcome upon discharge was significantly higher in the bridging IVT group compared to the direct MT group (34.6\% vs. $23.4 \%$, $P=0.047)$. In the bridging IVT group, 89.8\% (114/127) of the patients achieved successful reperfusion ( $\mathrm{mTICl} 2 b-3)$, compared to $83.4 \%(161 / 193)$ in the direct MT group $(P=0.110)$. In-hospital mortality rate was $19.7 \%$ in the bridging IVT group, compared to $26.7 \%$ in the direct MT group $(P=0.226)$. Few patients sustained hemorrhagic complications, and the rates of sICH in the two treatment groups were similar (5.0\% in bridging IVT vs. $4.4 \%$ in direct MT, $P=0.811$ ). Similarly, rates of SAH were comparable between both treatment groups (3.0\% vs. $3.6 \%, P=1.000$ ).

After adjustment for age, sex, NIHSS, and time from stroke onset to groin puncture in the multivariable model, the associ-

Table 4. Subgroup analysis comparing outcomes of bridging IVT vs. direct MT in basilar artery occlusion patients with underlying large-artery atherosclerosis $(n=101)$

\begin{tabular}{|c|c|c|c|c|c|}
\hline Outcome & Total $(n=101)$ & Bridging IVT $(n=43)$ & Direct MT $(n=58)$ & OR $(95 \% \mathrm{Cl})$ & $P$ \\
\hline 90-Day mRS & & & & $3.23(1.26-8.28)$ & 0.013 \\
\hline 0-3 (favorable) & $25(24.8)$ & 16 (37.2) & $9(15.5)$ & & \\
\hline 4-6 (unfavorable) & 76 (75.2) & 27 (62.8) & $49(84.5)$ & & \\
\hline 90-Day mRS & & & & $4.10(1.32-12.75)$ & 0.010 \\
\hline $0-2$ (good) & 17 (16.9) & $12(27.9)$ & $5(8.6)$ & & \\
\hline 3-6 (poor) & $84(83.2)$ & $31(72.1)$ & $53(91.4)$ & & \\
\hline Discharge mRS & & & & 6.28 (2.08-18.98) & $<0.001$ \\
\hline $0-3$ (good) & 21 (20.8) & $16(37.2)$ & $5(8.6)$ & & \\
\hline 4-6 (poor) & $80(79.2)$ & 27 (72.8) & $53(91.4)$ & & \\
\hline In-hospital mortality & & & & $0.34(0.13-0.90)$ & 0.027 \\
\hline Survival & $73(72.3)$ & 36 (83.7) & $37(63.8)$ & & \\
\hline Death & $28(27.7)$ & $7(16.3)$ & $21(36.2)$ & & \\
\hline $\mathrm{mTICl}$ post-treatment & & & & $2.07(0.51-8.33)$ & 0.346 \\
\hline $0-2 a$ (poor) & 11 (10.9) & $3(7.0)$ & $8(13.8)$ & & \\
\hline $2 b-3$ (good) & $90(89.1)$ & 40 (93.0) & $50(86.2)$ & & \\
\hline $\mathrm{mTICl}$ post-treatment & & & & $1.89(0.81-4.43)$ & 0.117 \\
\hline $0-2 b$ (poor) & $37(36.6)$ & $12(27.9)$ & $25(43.1)$ & & \\
\hline $2 c-3$ (good) & $64(63.4)$ & $31(72.1)$ & $33(56.9)$ & & \\
\hline sICH 24-hour post-intervention & $7(6.9)$ & $3(7.0)$ & $4(6.9)$ & $1.01(0.22-4.78)$ & 1.000 \\
\hline Subarachnoid hemorrhage & $2(2.0)$ & $1(2.3)$ & $1(1.7)$ & 1.36 (0.08-22.33) & 1.000 \\
\hline
\end{tabular}

Values are presented as number (\%).

$\mathrm{IVT}$, intravenous thrombolysis; $\mathrm{MT}$, mechanical thrombectomy; $\mathrm{OR}$, odds ratio; $\mathrm{Cl}$, confidence interval; $\mathrm{mRS}$, modified Rankin Scale; mTICl, modified Thrombolysis in Cerebral Infarction; $\mathrm{SICH}$, symptomatic intracranial hemorrhage. 
ation between bridging IVT and favorable functional outcome upon discharge lost significance $(\mathrm{OR}, 1.60 ; 95 \% \mathrm{Cl}, 0.82$ to 3.10; $P=0.169$ ) (Table 3). Similarly, bridging IVT was not significantly associated with the other outcomes upon multivariable adjustment (Table 3 ).

\section{Subgroup analysis}

A significant treatment effect of bridging IVT was observed in the subgroup of patients who had underlying LAA (Table 4). In this subgroup, patients treated with bridging IVT compared to direct MT had a high rate of favorable functional outcome at 90 days $(37.2 \%$ vs. $15.5 \%, P=0.013)$. A similar trend was observed with regard to other functional outcome measures, mRS $0-2$ at 90 days $(27.9 \%$ vs. $8.6 \%, P=0.010)$ and $\mathrm{mRS} 0-3$ at discharge $(37.2 \%$ vs. $8.6 \%, P<0.001)$. In-hospital mortality was also lower in the bridging IVT group compared to the direct MT group ( $16.3 \%$ vs. $36.2 \%, P=0.027$ ). Rates of successful reperfusion, sICH and SAH were similar between the bridging IVT and direct MT groups (Table 4). On ordinal analyses, bridging IVT was associated with a favorable shift in the $\mathrm{mRS}$ outcomes in BAO patients with LAA $(O R, 2.99 ; 95 \% \mathrm{Cl}, 1.42$ to 6.28 ; $P=0.004$ ) (Figure 1).

In all other subgroup analyses performed, there were no significant differences in the rates of favorable functional outcome at 90 days between the bridging IVT group and the direct
MT group (Supplementary Table 2).

\section{Discussion}

In this study, we did not observe a significant improvement in outcomes for BAO AIS patients who received combined bridging IVT and MT, compared to direct MT alone. However in a subgroup analysis, we found that bridging IVT prior to MT was associated with better functional outcomes in patients with BAO due to underlying LAA.

In the recently published Basilar Artery International Cooperation Study (BASICS) trial, $44.2 \%$ of patients with best medical management in addition to MT achieved favorable mRS scores (mRS 0-3) at 90 days post-discharge compared to $37.7 \%$ of those managed with best medical treatment alone. ${ }^{32}$ In our current study, we report a lower rate of favorable functional outcome of $39.4 \%$ and $34.4 \%$ in the bridging and direct MT group respectively. The discrepancies in the rates of $\mathrm{mRS}$ at 90 days is likely due to this being a real-world study, in the absence of a controlled clinical trial setting of ideal patient selection. Nonetheless, the rates of favorable functional outcomes in our study is similar to other large real-world registries, including the recently published EVT for Acute Basilar Artery Occlusion Study (BASILAR) study (32\% of patients achieved mRS $0-3$ in that study). ${ }^{33}$

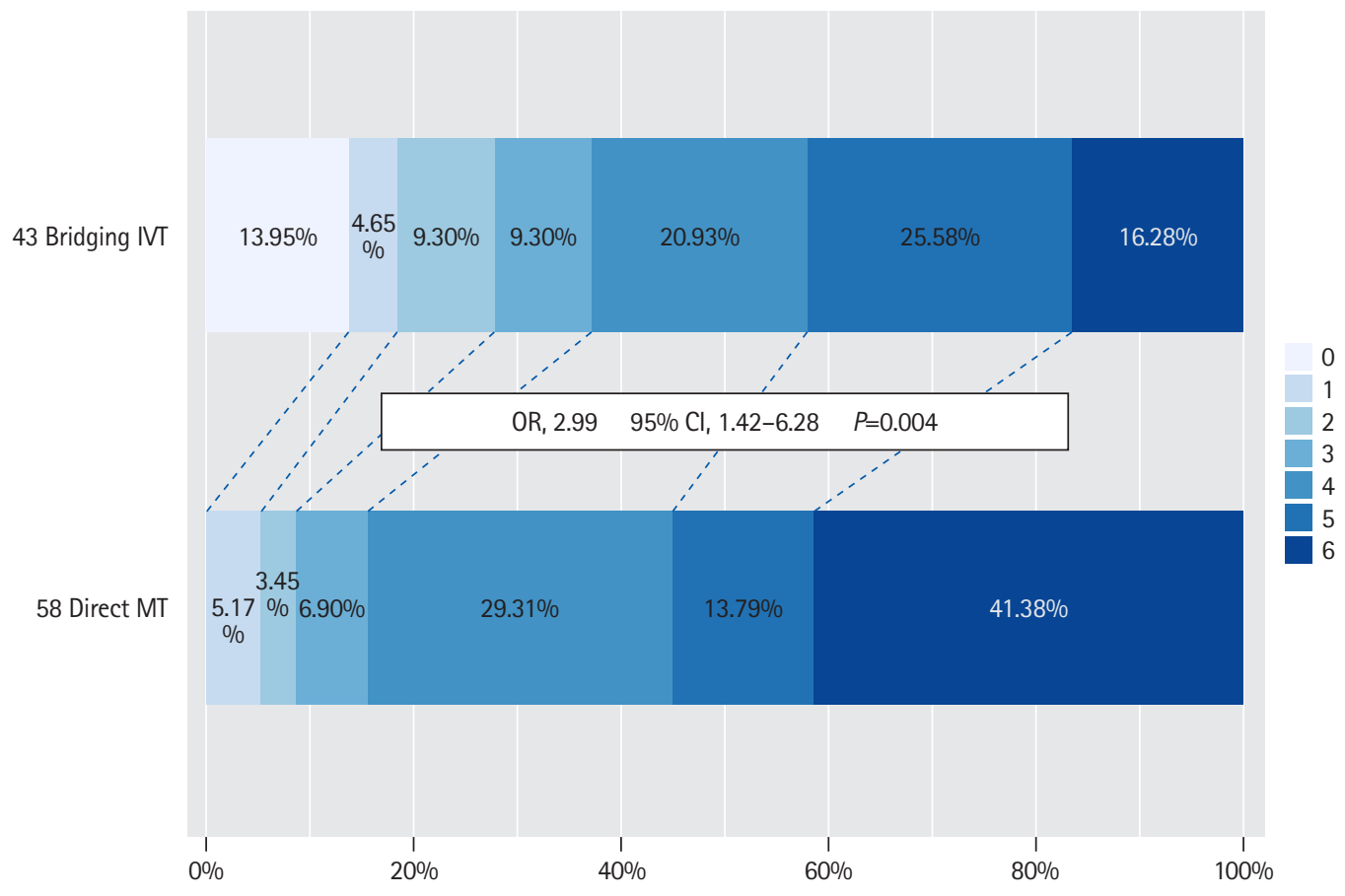

Figure 1. Ordinal shift analysis comparing 90-day modified Rankin Scale scores in patients with basilar artery occlusion acute ischemic stroke and underlying large-artery atherosclerosis who underwent bridging intravenous thrombolysis (IVT) versus direct mechanical thrombectomy (MT). OR, odds ratio; $\mathrm{Cl}$, confidence interval. 
Currently, there is a paucity of evidence comparing direct MT and bridging IVT in the treatment of acute $\mathrm{BAO}$, with no consensus on the optimal clinical management of this condition. ${ }^{19-22,25}$ Arguments in favor of bridging IVT include early reperfusion and the recanalization of thrombi in microvasculature that are inaccessible to thrombectomy devices. ${ }^{16}$ Arguments opposing bridging IVT include the risks of intracranial hemorrhage, thrombus fragmentation and distal embolization. ${ }^{34}$ Moreover, IVT is less effective in patients with LVO with a large thrombus burden as it is challenging for thrombolysis to penetrate the core of large thrombi and the surface areas exposed is less. ${ }^{35-37}$ Therefore the purported minimal benefit of bridging IVT is outweighed by the risks associated with hemorrhagic complications. ${ }^{34}$

Recent trials also support our findings that bridging IVT does not improve long-term patient outcomes. ${ }^{38,39}$ Upcoming randomized controlled trials exploring the efficacy of bridging IVT compared to direct MT in AISs include The Multicenter Randomized CLinical trial of Endovascular treatment for Acute ischemic stroke in the Netherlands (MR CLEAN-NO IV), ${ }^{40}$ Solitaire $^{\mathrm{TM}}$ With the Intention For Thrombectomy Plus Intravenous t-PA Versus DIRECT Solitaire ${ }^{\mathrm{TM}}$ Stent-retriever Thrombectomy in Acute Anterior Circulation Stroke (SWIFT DIRECT), ${ }^{41}$ and Randomized Controlled Trial of DIRECT Endovascular Clot Retrieval Versus Standard Bridging Thrombolysis With Endovascular Clot Retrieval Within 4.5 Hours of Stroke Onset (DIRECT SAFE). ${ }^{42}$ The caveat is that these studies are not specific to BAO ischemic stroke. Accordingly, the equipoise to whether bridging thrombolysis provides additional benefits over direct MT should be conclusively resolved by future larger randomized controlled studies or meta-analysis of trials, with the hopes of optimising treatment for BAO ischemic stroke. In our study, bridging IVT prior to MT was associated with better short term functional outcomes upon discharge. However, on multivariable analysis, the effect of bridging IVT on discharge mRS scores had lost its significance. This finding has been reported by similar studies regarding the management of LVO AIS wherein patients in the bridging IVT group had an early advantage compared to their counterparts who underwent direct $\mathrm{MT}^{23,43}$ A postulated theory is that systemic thrombolysis lyses the remaining small thrombi and distal emboli, leading to an early advantage in functional outcomes in the group of patients who underwent bridging $\mathrm{IVT}^{34}$ At the time of long-term follow-up, this effect may not be captured by the mRS score that focuses more on motor deficits than on cognitive outcomes.

Interestingly, our study found that patients with LAA appeared to benefit from bridging IVT. In LAA, the existing narrowing of cerebral vessels triggers the formation of collateral vessels over time. ${ }^{44}$ It is postulated that the thrombolytic agent flows through collateral vessels as well, allowing it to reach the distal end of the thrombus more effectively, ${ }^{44}$ in comparison to embolic strokes and strokes of other aetiologies where the collateral circulation development is more limited. ${ }^{45,46}$ Large scale studies have shown that the number of available connections to the anterior circulation is an independent predictor of good clinical outcome in BA0. ${ }^{46,47}$ Additionally, in LAA strokes with intracranial stenosis, it can be technically challenging to remove the thrombus via MT due to the narrowed lumen of cerebral vessels, with the potential to re-occlude. ${ }^{46}$ Administration of IVT potentially softens or dissolves the thrombus, facilitating more effective MT and better patient outcomes. ${ }^{34}$ Therefore, patients with LAA aetiology of BAO ischemic stroke could potentially be candidates for bridging IVT. Notably, the majority of patients categorised with LAA had intracranial atherosclerotic disease. ${ }^{36}$ Nonetheless, larger randomized studies are needed to validate our findings.

The limitations of our study stem from its retrospective non-randomized nature. Firstly, a difference in stroke onset to groin puncture time was noted between the bridging thrombolysis group and direct MT group, which was adjusted for in the multivariable analyses. Secondly, treatment allocations were made at the discretion of the treating stroke neurologist and neurointerventionist. This may introduce selection bias as different centers may have had different clinical practices. In our study cohort, $76.4 \%$ of bridging IVT $(n=97 / 127)$ patients and around half of the patients in the direct MT group (50.3\%; $n=98 / 195$ ) had onset to groin puncture time within 4.5 hours. This reflects a large proportion of patients in the direct MT group that presented early enough to be treated with bridging IVT; however, the attending stroke neurologist elected to forgo bridging IVT. Unfortunately, we did not collect the specific reasons for not administering IVT in this retrospective study. Nonetheless, our findings are reflective of real-world treatment paradigms as they reflect the same inconsistencies observed in treatment allocations by different physicians even within the same center, facilitating our improved understanding of the appropriate therapeutic strategies. Finally, the lack of statistical significance reported for the primary study outcome could be a function and limitation of the moderate sample size. Our study included a total of 322 acute BAO patients who underwent MT, which is a respectable cohort size in view that the procedure is less commonly performed for BAO in comparison to anterior circulation LVO. This sample size is also comparable to recently published bridging IVT versus direct MT randomized controlled trials for anterior circulation LVO. ${ }^{38,39}$ 


\section{Conclusions}

In patients with AIS due to BAO, bridging IVT was not associated with improved functional outcomes at 90-day. However, in patients with underlying LAA, a significant treatment effect of bridging IVT was observed. The equipoise to whether bridging IVT provides additional benefits over direct MT should be resolved in future randomized controlled studies.

\section{Supplementary materials}

Supplementary materials related to this article can be found online at https://doi.org/10.5853/jos.2021.02082.

\section{Disclosure}

The authors have no financial conflicts of interest.

\section{References}

1. Lindsberg PJ, Mattle HP. Therapy of basilar artery occlusion: a systematic analysis comparing intra-arterial and intravenous thrombolysis. Stroke 2006;37:922-928.

2. Schonewille WJ, Algra A, Serena J, Molina CA, Kappelle $\sqcup$. Outcome in patients with basilar artery occlusion treated conventionally. J Neurol Neurosurg Psychiatry 2005;76:12381241.

3. Baird TA, Muir KW, Bone I. Basilar artery occlusion. Neurocrit Care 2004;1:319-329.

4. Berkhemer OA, Fransen PS, Beumer D, van den Berg LA, Lingsma $\mathrm{HF}$, Yoo $\mathrm{A}$, et al. A randomized trial of intraarterial treatment for acute ischemic stroke. N Engl J Med 2015;372:11-20.

5. Campbell BC, Mitchell PJ, Kleinig TJ, Dewey HM, Churilov L, Yassi $N_{1}$ et al. Endovascular therapy for ischemic stroke with perfusion-imaging selection. N Engl J Med 2015;372:10091018.

6. Goyal M, Demchuk AM, Menon BK, Eesa M, Rempel JL, Thornton J, et al. Randomized assessment of rapid endovascular treatment of ischemic stroke. N Engl J Med 2015;372: 1019-1030.

7. Goyal M, Menon BK, van Zwam WH, Dippel DW, Mitchell PJ, Demchuk AM, et al. Endovascular thrombectomy after large-vessel ischaemic stroke: a meta-analysis of individual patient data from five randomised trials. Lancet 2016;387: 1723-1731.

8. Jovin TG, Chamorro A, Cobo E, de Miquel MA, Molina CA, Rovira $A$, et al. Thrombectomy within 8 hours after symptom onset in ischemic stroke. N Engl J Med 2015;372:2296-2306.
9. Saver JL, Goyal M, Bonafe A, Diener HC, Levy El, Pereira VM, et al. Stent-retriever thrombectomy after intravenous t-PA vs. t-PA alone in stroke. N Engl J Med 2015;372:2285-2295.

10. Singer OC, Berkefeld J, Nolte $\mathrm{CH}$, Bohner G, Haring HP, Trenkler J, et al. Mechanical recanalization in basilar artery occlusion: the ENDOSTROKE study. Ann Neurol 2015;77:415-424.

11. Jauch EC, Saver JL, Adams HP Jr, Bruno A, Connors JJ, Demaerschalk $B M$, et al. Guidelines for the early management of patients with acute ischemic stroke: a guideline for healthcare professionals from the American Heart Association/American Stroke Association. Stroke 2013;44:870-947.

12. Katsanos AH, Malhotra K, Goyal N, Arthur A, Schellinger PD,

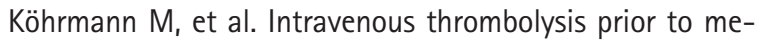
chanical thrombectomy in large vessel occlusions. Ann Neurol 2019;86:395-406.

13. Mazighi M, Meseguer $E_{1}$ Labreuche J, Amarenco P. Bridging therapy in acute ischemic stroke: a systematic review and meta-analysis. Stroke 2012;43:1302-1308.

14. Mazighi M, Serfaty JM, Labreuche J, Laissy JP, Meseguer E, Lavallée PC, et al. Comparison of intravenous alteplase with a combined intravenous-endovascular approach in patients with stroke and confirmed arterial occlusion (RECANALISE study): a prospective cohort study. Lancet Neurol 2009;8:802809.

15. Turc G, Bhogal P, Fischer U, Khatri P, Lobotesis K, Mazighi M, et al. European Stroke Organisation (ESO)-European Society for Minimally Invasive Neurological Therapy (ESMINT) guidelines on mechanical thrombectomy in acute ischaemic stroke: endorsed by Stroke Alliance for Europe (SAFE). Eur Stroke J 2019;4:6-12.

16. Desilles JP, Loyau S, Syvannarath V, Gonzalez-Valcarcel J, Cantier $M$, Louedec $L$, et al. Alteplase reduces downstream microvascular thrombosis and improves the benefit of large artery recanalization in stroke. Stroke 2015;46:3241-3248.

17. Seners P, Turc G, Maïer B, Mas JL, Oppenheim C, Baron JC. Incidence and predictors of early recanalization after intravenous thrombolysis: a systematic review and meta-analysis. Stroke 2016;47:2409-2412.

18. Tsivgoulis $G$, Katsanos $A H$, Schellinger PD, Köhrmann M, Varelas $P$, Magoufis $G$, et al. Successful reperfusion with intravenous thrombolysis preceding mechanical thrombectomy in large-vessel occlusions. Stroke 2018;49:232-235.

19. Abilleira S, Ribera $A$, Cardona $P$, Rubiera $M$, López-Cancio $E$, Amaro $S$, et al. Outcomes after direct thrombectomy or combined intravenous and endovascular treatment are not different. Stroke 2017;48:375-378.

20. Bellwald $S$, Weber R, Dobrocky $T$, Nordmeyer H, Jung $S$, Hadisurya J, et al. Direct mechanical intervention versus bridging 
therapy in stroke patients eligible for intravenous thrombolysis: a pooled analysis of 2 registries. Stroke 2017;48:32823288.

21. Coutinho JM, Liebeskind DS, Slater LA, Nogueira RG, Clark W, Dávalos $A$, et al. Combined intravenous thrombolysis and thrombectomy vs thrombectomy alone for acute ischemic stroke: a pooled analysis of the SWIFT and STAR studies. JAMA Neurol 2017;74:268-274.

22. Kaesmacher J, Mordasini P, Arnold M, López-Cancio E, Cerdá $\mathrm{N}$, Boeckh-Behrens T, et al. Direct mechanical thrombectomy in IPA-ineligible and -eligible patients versus the bridging approach: a meta-analysis. J Neurointerv Surg 2019;11:20-27.

23. Maingard J, Shvarts $Y$, Motyer R, Thijs V, Brennan P, O'Hare $A$, et al. Outcomes of endovascular thrombectomy with and without bridging thrombolysis for acute large vessel occlusion ischaemic stroke. Intern Med J 2019;49:345-351.

24. Seners $P$, Dargazanli $C$, Piotin $M$, Sablot $D$, Bracard $S$, Niclot $P$, et al. Intended bridging therapy or intravenous thrombolysis alone in minor stroke with basilar artery occlusion. Stroke 2021;52:699-702.

25. Mistry EA, Mistry AM, Nakawah MO, Chitale RV, James RF, Volpi JJ, et al. Mechanical thrombectomy outcomes with and without intravenous thrombolysis in stroke patients: a meta-analysis. Stroke 2017;48:2450-2456.

26. Powers WJ, Rabinstein AA, Ackerson T, Adeoye OM, Bam-

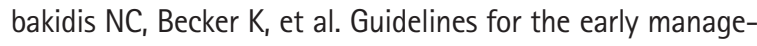
ment of patients with acute ischemic stroke: 2019 update to the 2018 guidelines for the early management of acute ischemic stroke: a guideline for healthcare professionals from the American Heart Association/American Stroke Association. Stroke 2019;50:e344-e418.

27. Sparaco M. Basilar artery occlusion: clinical management and therapy. Clin Manag Issues 2018;12:67-76.

28. Adams HP Jr, Bendixen BH, Kappelle $\sqcup$, Biller J, Love BB, Gordon $D L$, et al. Classification of subtype of acute ischemic stroke: definitions for use in a multicenter clinical trial: TOAST. Trial of Org 10172 in Acute Stroke Treatment. Stroke 1993;24: 35-41.

29. Dargazanli C, Fahed R, Blanc R, Gory B, Labreuche J, Duhamel $A_{1}$ et al. Modified thrombolysis in cerebral infarction $2 \mathrm{C} /$ thrombolysis in cerebral infarction 3 reperfusion should be the aim of mechanical thrombectomy: insights from the ASTER Trial (Contact Aspiration Versus Stent Retriever for Successful Revascularization). Stroke 2018;49:1189-1196.

30. Jansen IG, Mulder MJ, Goldhoorn RB; MR CLEAN Registry investigators. Endovascular treatment for acute ischaemic stroke in routine clinical practice: prospective, observational cohort study (MR CLEAN Registry). BMJ 2018;360:k949.
31. Wahlgren $N$, Ahmed N, Dávalos $A$, Hacke W, Millán $M$, Muir $\mathrm{K}$, et al. Thrombolysis with alteplase 3-4.5 $\mathrm{h}$ after acute ischaemic stroke (SITS-ISTR): an observational study. Lancet 2008;372:1303-1309.

32. Schonewille W. A randomized acute stroke trial of endovascular therapy in acute basilar artery occlusion. European Stroke Organisation (ESO)-World Stroke Organization (WSO); 2020 Nov 7-9; Webinar. https://eso-wso-conference.org/ eso-wso-may-webinar.

33. Writing Group for the BASILAR Group, Zi W, Oiu Z, Wu D, Li

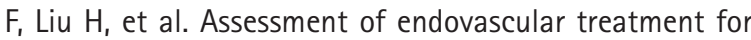
acute basilar artery occlusion via a nationwide prospective registry. JAMA Neurol 2020;77:561-573.

34. Fischer U, Kaesmacher J, Mendes Pereira V, Chapot R, Siddiqui $A H$, Froehler MT, et al. Direct mechanical thrombectomy versus combined intravenous and mechanical thrombectomy in large-artery anterior circulation stroke: a topical review. Stroke 2017;48:2912-2918.

35. Riedel $\mathrm{CH}$, Zimmermann $P$, Jensen-Kondering $U$, Stingele $R$, Deuschl G, Jansen 0 . The importance of size: successful recanalization by intravenous thrombolysis in acute anterior stroke depends on thrombus length. Stroke 2011;42:1775-1777.

36. Sun $X$, Tong $X$, Gao F, Lao H, Miao Z. Endovascular treatment for acute basilar artery occlusion: a single center retrospective observational study. BMC Neurol 2019;19:315.

37. Yang $P$, Zhang $Y$, Zhang L, Zhang Y, Treurniet KM, Chen $W$, et al. Endovascular thrombectomy with or without intravenous alteplase in acute stroke. N Engl J Med 2020;382:1981-1993.

38. Suzuki K, Matsumaru Y, Takeuchi M, Morimoto M, Kanazawa $R$, Takayama $Y$, et al. Effect of mechanical thrombectomy without vs with intravenous thrombolysis on functional outcome among patients with acute ischemic stroke: the SKIP randomized clinical trial. JAMA 2021;325:244-253.

39. Zi W, Qiu Z, Li F, Sang H, Wu D, Luo W, et al. Effect of endovascular treatment alone vs intravenous alteplase plus endovascular treatment on functional independence in patients with acute ischemic stroke: the DEVT randomized clinical trial. JAMA 2021;325:234-243.

40. Treurniet KM, LeCouffe NE, Kappelhof M, Emmer BJ, van Es $A C$, Boiten J, et al. MR CLEAN-NO IV: intravenous treatment followed by endovascular treatment versus direct endovascular treatment for acute ischemic stroke caused by a proximal intracranial occlusion-study protocol for a randomized clinical trial. Trials 2021;22:141.

41. Bridging Thrombolysis Versus Direct Mechanical Thrombectomy in Acute Ischemic Stroke (SWIFT DIRECT). ClinicalTrials. gov. https://clinicaltrials.gov/ct2/show/NCT03192332. 2021. Accessed November 4, 2021. 
42. DIRECT-SAFE: A Randomized Controlled Trial of DIRECT Endovascular Clot Retrieval Versus Standard Bridging Thrombolysis With Endovascular Clot Retrieval. ClinicalTrials.gov. https://clinicaltrials.gov/ct2/show/NCT03494920. 2021. Accessed November 4, 2021.

43. Chalos V, LeCouffe NE, Uyttenboogaart M, Lingsma HF,

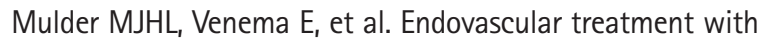
or without prior intravenous alteplase for acute ischemic stroke. J Am Heart Assoc 2019;8:e011592.

44. Malhotra K, Liebeskind DS. Collaterals in ischemic stroke. Brain Hemorrhages 2020;1:6-12.

45. Haussen DC, Bouslama M, Dehkharghanii S, Bowen M, Fag- gard J, Grossberg JA, et al. Enhanced collaterals in patients with large vessel stroke from intracranial atherosclerosis: an automated CT perfusion-based study. Stroke 2017;48(Suppl 1):AWMP23.

46. Liebeskind DS, Cotsonis GA, Saver JL, Lynn MJ, Turan TN, Cloft $\mathrm{HJ}$, et al. Collaterals dramatically alter stroke risk in intracranial atherosclerosis. Ann Neurol 2011;69:963-974.

47. Maus V, Kalkan A, Kabbasch C, Abdullayev N, Stetefeld H, Barnikol UB, et al. Mechanical thrombectomy in basilar artery occlusion: presence of bilateral posterior communicating arteries is a predictor of favorable clinical outcome. Clin Neuroradiol 2019;29:153-160. 
Supplementary Table 1. Participating study institutions across five countries

\begin{tabular}{ll}
\hline Germany & University Hospital Magdeburg \\
& University Medical Center Hamburg-Eppendorf \\
& University Hospital RWTH Aachen \\
& Hospital Bremen-Mitte \\
United Kingdom & The Royal London Hospital \\
Taiwan & Linkou Chang Gung Memorial Hospital \\
Sweden & Karolinska Institutet \\
Singapore & National University Hospital \\
\hline
\end{tabular}

Supplementary Table 2. Comparing 90-day functional outcomes of bridging IVT vs. direct MT in subgroup analyses

\begin{tabular}{|c|c|c|c|c|c|}
\hline Outcome & Total & Bridging IVT & Direct MT & OR $(95 \% \mathrm{Cl})$ & $P$ \\
\hline LAA 90-Day mRS & & & & $3.23(1.26-8.28)$ & 0.013 \\
\hline $0-3$ (good) & $25(24.8)$ & $16(37.2)$ & $9(15.5)$ & & \\
\hline 4-6 (poor) & $76(75.2)$ & $27(62.8)$ & $49(84.5)$ & & \\
\hline Non-LAA 90-day mRS & & & & $0.93(0.53-1.61)$ & 0.785 \\
\hline $0-3$ (good) & $92(41.6)$ & $34(40.5)$ & $58(42.3)$ & & \\
\hline 4-6 (poor) & $129(58.4)$ & $50(59.5)$ & $79(57.7)$ & & \\
\hline Young ( $\leq 75$ years) 90-day mRS & & & & $1.22(0.69-2.16)$ & 0.486 \\
\hline $0-3$ (good) & $87(41.0)$ & $34(44.2)$ & $53(39.3)$ & & \\
\hline 4-6 (poor) & $125(59.0)$ & $43(55.8)$ & $82(60.7)$ & & \\
\hline Old ( $>75$ years) 90-day mRS & & & & $1.55(0.67-3.59)$ & 0.310 \\
\hline $0-3$ (good) & $30(27.3)$ & $16(32.0)$ & $14(23.3)$ & & \\
\hline 4-6 (poor) & $80(72.7)$ & $24(68.0)$ & $46(76.7)$ & & \\
\hline Male 90-day mRS & & & & $1.30(0.72-2.36)$ & 0.384 \\
\hline $0-3$ (good) & $71(34.5)$ & $28(38.4)$ & $43(32.3)$ & & \\
\hline 4-6 (poor) & $135(65.5)$ & $45(61.6)$ & $90(67.7)$ & & \\
\hline Female 90-day mRS & & & & $1.09(0.52-2.29)$ & 0.823 \\
\hline $0-3$ (good) & $46(39.7)$ & $22(40.7)$ & $24(38.7)$ & & \\
\hline 4-6 (poor) & $70(60.3)$ & $32(59.3)$ & 38 (61.3) & & \\
\hline
\end{tabular}

Values are presented as number (\%).

IVT, intravenous thrombolysis; MT, mechanical thrombectomy; OR, odds ratio; $\mathrm{Cl}$, confidence interval; LAA, large-artery atherosclerosis; mRS, modified Rankin Scale. 